\title{
Predictive Control of Systems with Fast Dynamics Using Computational Reduction Based on Feedback Control Information
}

\author{
Tomáš Barot, Marek Kubalcik \\ Department of Process Control, Tomas Bata University in Zlín, Faculty of Applied \\ Informatics, nám. T. G. Masaryka 5555, 76001 Zlín, Czech Republic \\ \{barot, kubalcik\}@fai.utb.cz
}

\begin{abstract}
Predictive control is a method, which is suitable for control of linear discrete dynamical systems. However, control of systems with fast dynamics could be problematic using predictive control. The calculation of a predictive-control algorithm can exceed the sampling period. This situation occurs in case with higher prediction horizons and many constraints on variables in the predictive control. In this contribution, an improving of the classical approach is presented. The reduction of the computational time is performed using an analysis of steady states in the control. The presented approach is based on utilization of information from the feedback control. Then this information is applied in the control algorithm. Finally, the classical method is compared to the presented modification using the time analyses.
\end{abstract}

\section{Introduction}

Predictive control [1] is a modern method, which uses the principle based on computation on the future horizons in connection to the optimization problem solving. The future information about situation in the feedback control is predicted using a model of the identified system. The model is used as a predictor [2]. The unknown information is computed using optimization subsystem [3], in which are considered the requirements on the feedback control and all defined constraints.

In general, the algorithm of predictive control is suitable for many types of systems [4]. However, the category of systems with fast dynamics [5] could bring the problems. The disadvantage is based on the higher computational time of the algorithm with more difficult settings of the predictive controller, as can be seen in this contribution. These more demanding settings are the higher prediction horizons [6] and using of greater number of constraints [7] on variables in the control. The important assumption is solving prediction equations and the optimization problem in time of the sampling period. 
The approach, when optimization in connection to predictions is solved in each sampling period, is referred to be online. The optimization part included more significantly time-demanding parts than the predictor; therefore the research is focused on the optimizer.

The optimization task solved the minimization of the defined cost function, which is usually defined as the quadratic function. If the constraints are in the form of the linear inequalities [8], the task is defined as the quadratic programming problem [9]. The fast method used for this purposes is the Hildreth's method [10]. This algorithm uses the dual method [11] of the non-classical based extreme task [11].

For many types of controlled systems is this algorithm appropriate. However, the predictive control of systems with fast dynamics needs a modification of this principle. The modification [10] tries to remove all constraints in the optimization task in the current sampling period and then test the success of it. In this paper, the Hildreth's method with modification [10] is considered as the classical approach. The modified approach is based on using the information on the feedback control. If a steady state occured, the elimination of inactive constraints will be possible. The results are discussed in the final part of this contribution.

\section{Model of Controlled System in Predictive Controller}

In the predictive control, the controlled system can be mathematically described by a discrete transfer function (2) [5], which corresponds to the continuous representation [5] of this system (1) for given sampling period $T$. The linear variant of systems is considered in this paper. The future outputs of the system behaviour are determined using this model. The model is included in the predictor of the predictive controller.

In this paper, the systems with fast dynamics are considered. This category of systems is characterized by their roots with values in order of minus tens, in denominator of transfer function (1). In general, the sampling period is significantly low, in order of hundredths of a second.

$$
G(s)=\frac{\bar{b}_{1} s+\bar{b}_{2}}{\bar{a}_{1} s^{2}+\bar{a}_{2} s+\bar{a}_{3}}, G\left(z^{-1}\right)=\frac{b_{1} z^{-1}+b_{2} z^{-2}}{1+a_{1} z^{-1}+a_{2} z^{-2}}
$$

For the determination of future $N_{l} \ldots N_{2}$ outputs (4), the difference equation (3) is used. The equation (4) is based on CARIMA (Controlled Autoregressive Integrated Moving Average) model [10], which includes $N_{u}$ future increments of manipulated variable $\Delta u$ instead the direct value $u$. In predictive control, the parameters $N_{1}, N_{u}$ and $N_{2}$, determine the receding horizon window [6]. 


$$
\begin{gathered}
\left(1-z^{-1}\right)\left(1+a_{1} z^{-1}+a_{2} z^{-2}\right) y(k)=\left(b_{1} z^{-1}+b_{2} z^{-2}\right) \cdot \Delta u(k) \\
{\left[\begin{array}{c}
y\left(k+N_{1}\right) \\
\vdots \\
y\left(k+N_{2}\right)
\end{array}\right]=\boldsymbol{P} \cdot\left[\begin{array}{c}
y(k) \\
y(k-1) \\
y(k-2) \\
\Delta u(k-1)
\end{array}\right]+\boldsymbol{G} \cdot\left[\begin{array}{c}
\Delta u(k) \\
\Delta u(k+1) \\
\vdots \\
\Delta u\left(k+N_{u}-1\right)
\end{array}\right]}
\end{gathered}
$$

Matrices $\boldsymbol{P}$ and $\boldsymbol{G}$ contain coefficients of difference equations. They are determined recursively [10]. The part of the matrix equation (4) with matrix $\boldsymbol{P}$ includes the information from the past; whereas, the part with matrix $\boldsymbol{G}$ is related to the predicted situation. The future information is determined using optimization subsystem.

\section{Control Law of Predictive Control}

The control law has atypical form (5) in predictive control. Equation (5) is the solving of optimization task, where the sequence of $N_{u}$ values of future values of manipulated-variable increments (the vector $\Delta \boldsymbol{u}$ ) is determined. In this case, the quadratic cost function with constraints is used (6). This problem is then a quadratic programming task [9] and should be algorithmically solved by the fast Hildreth's method [10].

$$
\Delta \boldsymbol{u}=\arg \left[\min \left\{\frac{1}{2} \Delta \boldsymbol{u}^{T} . \boldsymbol{H} \cdot \Delta \boldsymbol{u}+\boldsymbol{b}^{T} . \Delta \boldsymbol{u}\right\}\right], \boldsymbol{M} . \Delta \boldsymbol{u} \leq \boldsymbol{K}
$$

The matrices $\boldsymbol{P}$ and $\boldsymbol{G}$, from the predictor, are utilized for determination of vector $\boldsymbol{b}$ (7) and Hessian matrix $\boldsymbol{H}(8)$, where $\boldsymbol{I}$ is a unit matrix with dimension $N_{u}$ and $w$ is reference signal. Finally, the form of the cost function expresses the requirements on the feedback control [6].

$$
\begin{gathered}
\boldsymbol{b}=2\left(\boldsymbol{P}\left[\begin{array}{llll}
y(k) & y(k-1) & y(k-2) & \Delta u(k-1)
\end{array}\right]^{T}-\right. \\
-\left[\begin{array}{ll}
w\left(k+N_{1}\right) \cdots w\left(k+N_{2}\right) & \cdots
\end{array}\right)^{T} \boldsymbol{G} \\
\boldsymbol{H}=2 .\left(\boldsymbol{G}^{\boldsymbol{T}} \cdot \boldsymbol{G}+\boldsymbol{I}\right)
\end{gathered}
$$


The form of matrices $\boldsymbol{M}$ and $\boldsymbol{K}$ can be constructed by rules, which can be seen in Table 1, where $\boldsymbol{I}$ is a unit matrix with dimension $N_{u}, \boldsymbol{T}$ is a lower-triangular matrix with dimension $N_{u}$ and $\boldsymbol{E}$ is an ones matrix. Each type of constraint has a form, which has been derived for the variable $\Delta \boldsymbol{u}$.

Table 1 Rules for building matrices $\boldsymbol{M}$ and $\boldsymbol{K}$ for types of restrictions on control.

\begin{tabular}{|c|c|c|}
\hline Restriction & $M$ & $\boldsymbol{K}$ \\
\hline$u_{\min }$ & $-T$ & $-\boldsymbol{E}^{N_{u}, 1} \cdot u_{\min }+\boldsymbol{E}^{N_{u}, 1} \cdot u(k-1)$ \\
\hline$u_{\max }$ & $T$ & $\boldsymbol{E}^{N_{u}, 1} \cdot u_{\max }-\boldsymbol{E}^{N_{u}, 1} . u(k-1)$ \\
\hline$y_{\min }$ & $-\boldsymbol{G}^{N_{2}-N_{1}, N}$ & $\begin{array}{c}\quad-\boldsymbol{E}^{N_{2}-N_{1}+1,1} y_{\text {min }}+ \\
+\boldsymbol{P} \cdot\left[\begin{array}{llll}y(k) & y(k-1) & y(k-2) & \Delta u(k-1)\end{array}\right]\end{array}$ \\
\hline$y_{\max }$ & $\boldsymbol{G}^{N_{2}-N_{1}, N_{u}}$ & 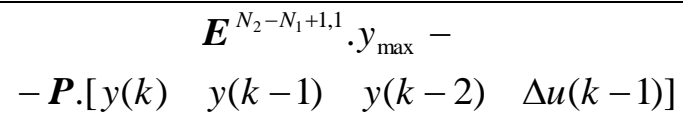 \\
\hline$\Delta u_{\min }$ & $-\boldsymbol{I}$ & $-\boldsymbol{E}^{N_{u}, 1} \cdot \Delta u_{\min }$ \\
\hline$\Delta u_{\max }$ & $I$ & $\boldsymbol{E}^{N_{u}, 1} \cdot \Delta u_{\max }$ \\
\hline
\end{tabular}

\section{Classical Method}

Subsystems of predictive controller - the predictor and the optimizer cooperate together by equations (5)-(6) and (4). The control law is enumerated in each sampling period in the predictive control. For higher setting of parameters in predictive controller, computations could be so time consuming, that they overload the time of sampling period. In case of controlled systems with fast dynamics, the higher value of parameter $N_{2}$ and the increasing number of constraints could cause a problem.

The modification of these cases was published in [10]. The main idea was to leave out all constraints (9) in computation of the optimization problem [9] by Hildreth's method.

$$
\Delta \boldsymbol{u}=-\boldsymbol{H}^{-1} \boldsymbol{b}
$$


If the result passes the constraints condition (6) retrospectively, it is supposed to be the final result of the task. The non-classical based extreme task will be transferred to the multidimensional-free-extreme problem without consideration of all constraints. The time complexity can be decreased by this approach. However; this method has one disadvantage. This reduction of steps in optimization algorithm can not be applied in each sampling period in predictive control. This reduction is not naturally possible in all cases. The aim is to decrease the computational time in all steps of the discrete control.

\section{Approach for Computation Reduction}

The modified approach removes constraints, when the classical method with result (9) by condition (6) is not successful. The constraints in predictive control can be left out in case of stabilization of variables in the feedback control, when value $y$ is steady. In this situation, all constraints can be tested for their reduction.

At first, the success of the classical method with constraints removing is tested in the optimization task. Otherwise; in steady state (10) in feedback control, the presented approach is applied and tested by (6). In other cases must be performed the whole optimization algorithm. The summary scheme can be seen in Fig. 1 and Fig.2.

$$
\begin{aligned}
& \forall\left\{\left|e\left(k+\left.i\right|_{k-1}\right)=w\left(k+\left.i\right|_{k-1}\right)-y\left(k+\left.i\right|_{k-1}\right)\right| \leq \varepsilon, i=\left\{N_{1}, \ldots, N_{2}\right\}\right\}, \\
& \varepsilon \approx 0, k \geq 1 \\
& \int M . \Delta u \leq K \rightarrow \text { (5) success of the classical method }
\end{aligned}
$$

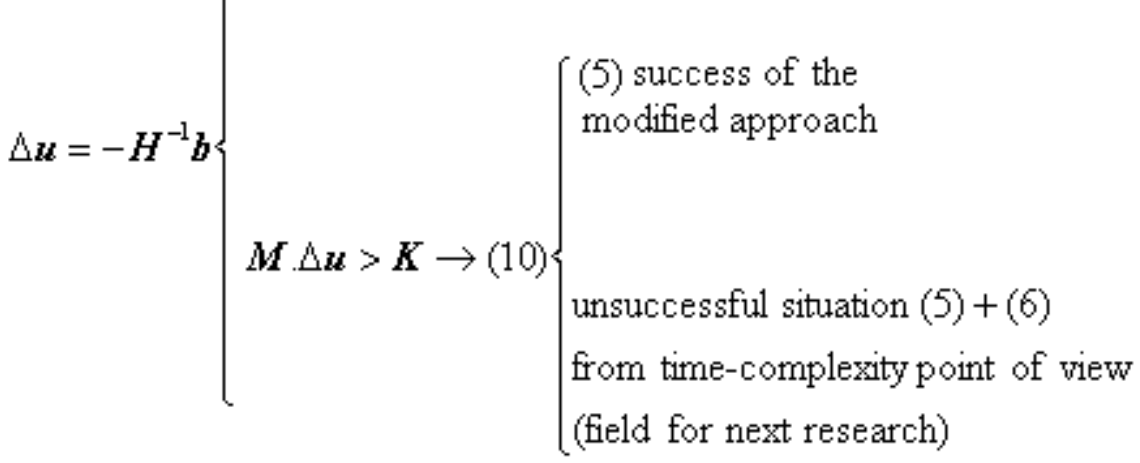

Fig. 1 Inclusion of Proposed Approach in Whole Optimization Strategy 


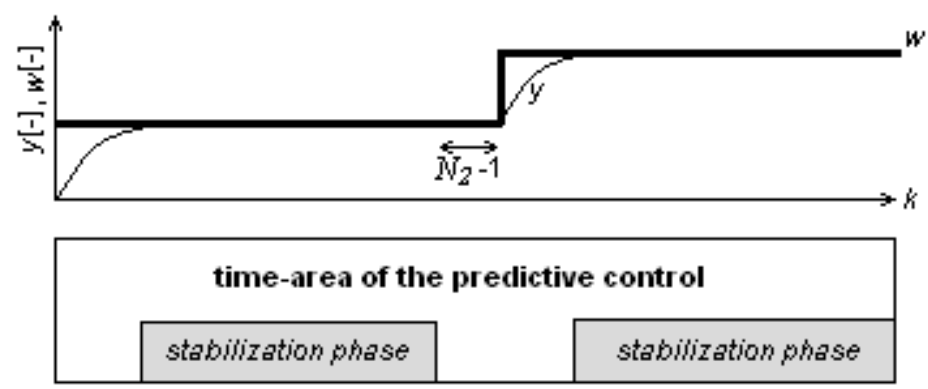

(9) is controlled by condition (6) ..... classical method

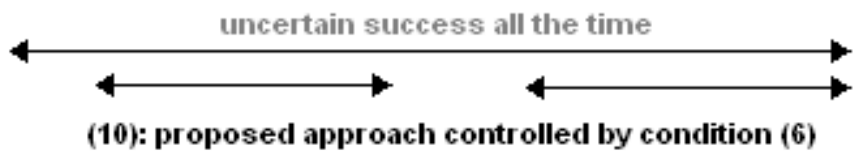

Fig. 2 Time-areas of Classical and Proposed Approaches

\section{Results}

Predictive control algorithm with optimization Hildreth's algorithm was realized in MATLAB environment. The time-measuring functions were implemented for the purposes of time analyses of presented approach in comparison to the classical method.

The controlled system (11) with fast dynamics is chosen and the corresponding discrete model (12) is determined for sampling period $T=0.05 \mathrm{~s}$. The roots of the denominator are -10 and -14 .

$$
\begin{gathered}
G(s)=\frac{s+350}{s^{2}+24 s+140} \\
G\left(z^{-1}\right)=\frac{0.324 z^{-1}+0.1712 z^{-2}}{1-1.103 z^{-1}+0.3012 z^{-2}}
\end{gathered}
$$

The setting of predictive controller is as follows:

- horizons:

$$
N_{l}=1, N_{u}=25, N_{2}=30
$$

- reference signal values: $w_{\min }=0.5, w_{\max }=1$

- constraints: $u_{\min }=0, u_{\max }=1, \Delta u_{\min }=0.02, \Delta u_{\max }=0.2, y_{\min }=0, y_{\max }=1$

The parameter $\varepsilon$ in (10) is equal to 0 in this simulation example. 
The simulation of the predictive control of system (12) can be seen in Fig. 3. On the axis $k$ are ordinates of the discrete control that respect the sampling period $T$.

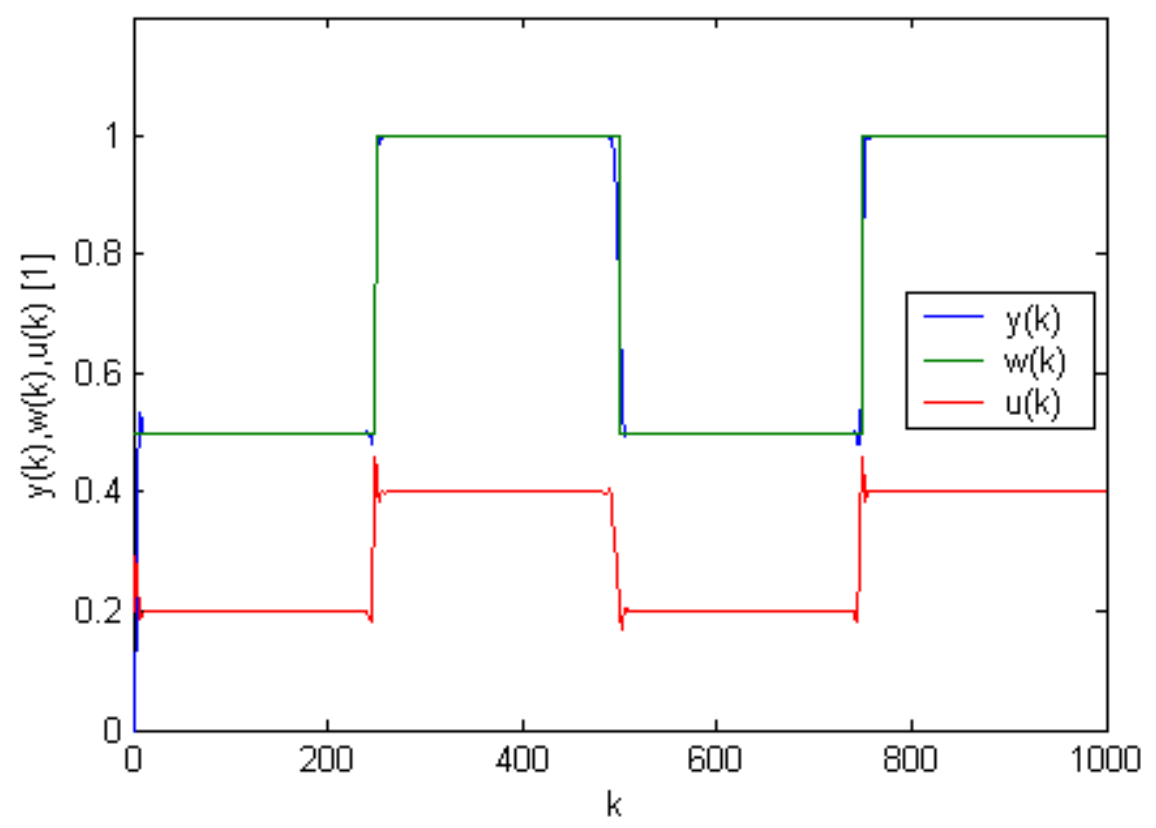

Fig. 3 Simulation of Predictive Control of System with Fast Dynamics

In Fig.4 and Fig.5, the time analysis can be seen. The time consuming operations of optimization subsystem were measured in MATLAB script using timemeasuring function. Figures show the time $T_{\mathrm{c}}$ that was needed for executing of the optimization algorithm in each sampling period.

At first it is displayed the analysis for the classical method (Fig.4) and then for application of combination of both approaches (the classical one together with the proposed one) (Fig.5). The predictive control was realized hundred times and the average results are displayed for all sampling times. 


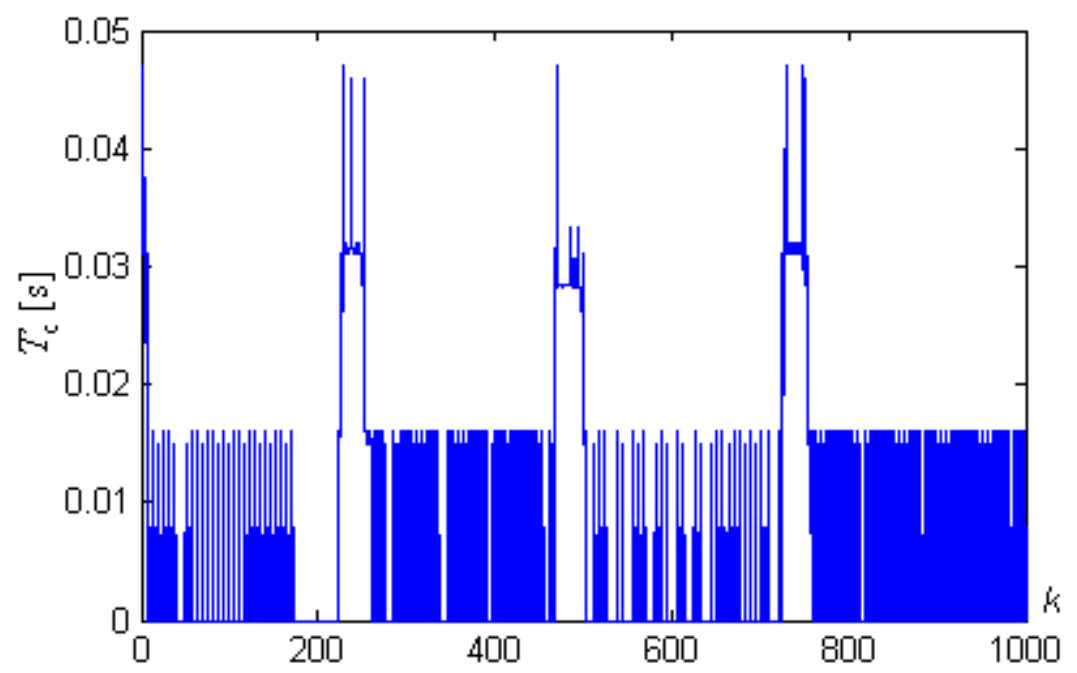

Fig. 4 Computing Time Analysis Only for Classical Method

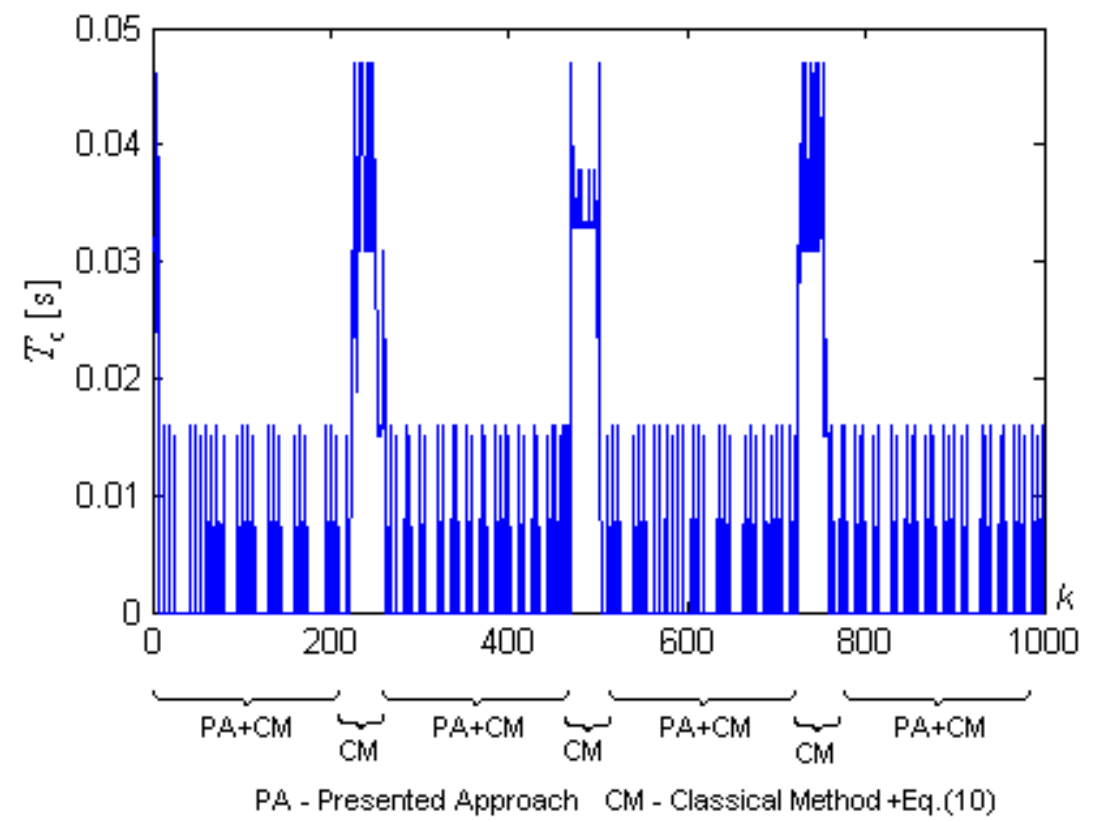

Fig. 5 Time Analysis for Proposed Approach Together with Classical Method 


\section{Conclusion}

The improving of time-consuming approach was designed and realized for the predictive control of systems with fast dynamics. For higher horizons and many applied constraints it is needed to decrease the computational time in control algorithm. Without this modification, the performing of the algorithm can overload the sampling period. The classical approach tests, if the multidimensional free extreme task is appropriate for the optimization task of quadratic programming with constraints. This method may not pass the constraints conditions in all sampling periods of predictive control. The proposed approach uses the information from the control. In this situation some constraints can be reduced. The time-analyses confirmed, that better results were achieved using the presented modification. However, it is needed to eliminate the computational time in each sampling period of predictive control. This can be investigated in the further research.

Acknowledgment: The article was realized with financial support of IGA, Tomas Bata University in Zlín, Faculty of Applied Informatics number IGA/FAI/2015/006.

\section{References}

1. E.F. Camacho and C. Bordons, Model Predictive Control, Springer, London, 2007.

2. Corriou, Jean-Pierre. Process control: theory and applications, London: Springer, 2004. ISBN 1-85233-776-1.

3. Rawlings, James B., and David Q. Mayne. Model Predictive Control: Theory and Design. Madison: Nob Hill Publishing, 2009. ISBN 978-0-9759377-0-9.

4. Kučera, Vladimír. Analysis and Design of Discrete Linear Control Systems. Praha: Nakladatelství Československé akademie věd, 1991. ISBN 80-200-0252-9.

5. BALÁTĚ, Jaroslav. Automatické řizení. 2. vyd. Praha: BEN - technická literatura, 2004. ISBN 80-7300-148-9 [in Czech].

6. W.H. Kwon, Receding horizon control: model predictive control for state models, London: Springer, 2005.

7. Huang, Sunan. Applied predictive control. London: Springer, 2002. ISBN 1852333383.

8. G.M. Lee, N.N. Tam, and N.D. Yen, Quadratic Programming and Affine Variational Inequalities: A Qualitative Study, Springer, 2005.

9. Z. Dostál, Optimal Quadratic Programming Algorithms: With Applications to Variational Inequalities, New York: Springer, 2009.

10. Wang, Liuping. Model Predictive Control System Design and Implementation Using MATLAB. London: Springer-Verlag Limited, 2009. ISBN 978-1-84882-330-3.D.G.

11. Luenberger, David G., and Yinyu YE. Linear and nonlinear programming. New York: Springer, 2008. ISBN 978-0-387-74502-2. 